\title{
IGNATOV'S THEOREM: AN ABBREVIATION OF THE PROOF OF ENGELEN, TOMMASSEN AND VERVAAT
}

\author{
L. C. G. ROGERS, ${ }^{*}$ University of Cambridge
}

The beautiful proof of Ignatov's theorem which appeared in Engelen et al. (1988) is obviously the correct way in which to prove this amazing result. The purpose of this note is to show that, by assembling ideas of Engelen et al. in a slightly different way, one can deduce the general case from the discrete case.

Recall the situation. $X_{1}, X_{2}, \cdots$ are i.i.d. real-valued random variables. The observation $X_{n}$ is a $k$-record value if $\sum_{i=1}^{n} I_{\left\{X_{i} \geq X_{n}\right\}}=k$, and the collection of all $k$-record values makes up a point process on $\mathbb{R}$, called the $k$-record process. Let us denote the $k$-record process by $\left(N_{x}^{k}\right)_{x \in \mathbb{R}}$, using the 'counting process' formulation $N_{x}^{k} \equiv$ no. of $k$-record values $\leqq x$. Ignatov's incredible result says that $N^{1}, N^{2}, \cdots$ are i.i.d. point processes and Engelen et al. prove this very quickly assuming that the distribution of $X_{1}$ is discrete (Section 2 of their paper).

To pass to the general case, we shall prove by a discretization argument that for any $K, M \in \mathbb{N}$, the restrictions to $(-\infty, M)$ of $N^{1}, \cdots, N^{K}$ are i.i.d., which is all that is needed. Consider the approximations $X_{i}^{n}$ to $X_{i}$ defined by $X_{i}^{n} \equiv 2^{-n}\left[2^{n} X_{i}\right]$, where [·] denotes the integer part. Then always $X_{i}-2^{-n}<X_{i}^{n} \leqq X_{i}$, and for the i.i.d. sequence $\left(X_{i}^{n}\right)_{i \in \mathbb{N}}$, Ignatov's theorem holds, since $X_{1}^{n}$ has a discrete distribution.

Let

$$
\begin{aligned}
\tau & \equiv \inf \left\{m: \sum_{j=1}^{n} I_{[M, \infty)}\left(X_{j}^{n}\right)=K\right\} \\
& \equiv \inf \left\{m: \sum_{j=1}^{n} I_{[M, \infty)}\left(X_{j}\right)=K\right\} .
\end{aligned}
$$

Evidently, it is impossible for any $X_{j}, j>\tau$, to be a $k$-record value, $k \leqq K$, except if $X_{j} \geqq M$. The same is true for the approximations $\left(X_{j}^{n}\right)$. Thus the restrictions to $(-\infty, M)$ of the $k$-record processes, $k=1, \cdots, K$, are known once $X_{1}, \cdots, X_{\tau}$ have been observed. Now while the $k$-record processes of $\left(X_{j}^{n}\right)_{j \in \mathbb{N}}$ are not easily related to the $k$-record processes of $\left(X_{j}\right)_{j \in \mathbb{N}}$ (since different $X_{j}$-values may get aliased into the same $X_{j}^{n}$-value, and may thereby get attributed to the wrong $k$-record process) what we can say is that for $n$ sufficiently large, the mesh $2^{-n} \mathbb{Z}$ will be fine enough to distinguish all different $X_{j}$-values, $j=1, \cdots, \tau$. Thus, almost surely, the restrictions to $(-\infty, M)$ of the $k$-record process of the $\left(X_{j}^{n}\right)$ converge (weakly) to the $k$-record processes of $\left(X_{j}\right)$, and the independence of $N^{1}, N^{2}, \cdots$ follows.

We note also that the common law of the $N^{i}$ can be easily identified, by taking some continuous $f \geqq 0$ supported in $(-M, M)$ and computing the Laplace functional $\phi(f) \equiv E \exp \left\{-\int f(x) N^{-1}(d x)\right\}$. If the distribution were discrete, $P\left(X_{1}=x_{j}\right)=\phi_{j}$, then elementary calculations give

$$
\phi(f)=\exp \left\{\sum_{j} \log \left(1-p_{j} f\left(x_{j}\right) / \bar{p}_{j}\right)\right\}
$$

where $\bar{p}_{j} \equiv \sum_{k \geqq j} p_{k}$. If $\phi_{n}$ is the Laplace functional of the $n$th approximation to $N^{1}$, then the

Received 16 May 1989; revision received 12 June 1989.

* Postal address: Statistical Laboratory, University of Cambridge, 16 Mill Lane, Cambridge CB2 1SB, UK. 
almost sure convergence of the 1-record processes implies that $\phi_{n}(f) \rightarrow \phi(f)$, characterising the (simplified Poisson) law of $N^{1}$.

\section{Reference}

Engelen, R., Tommassen, P., AND VervaAt, W. 1988) Ignatov's theorem; a new and short proof. $J$. Appl. Prob. 25A, 229-236. 